Article

\title{
Identifying Safety Training Resource Needs in the Cattle Feeding Industry in the Midwestern United States
}

\author{
Athena K. $\operatorname{Ramos}^{1, *(D)}$, Ellen Duysen ${ }^{2}$ and Aaron M. Yoder ${ }^{2} \mathbb{D}$ \\ 1 Center for Reducing Health Disparities, Department of Health Promotion, University of Nebraska Medical \\ Center, Omaha, NE 68198-4340, USA \\ 2 Central States Center for Agricultural Safety and Health, Department of Environmental, Agricultural and \\ Occupational Health, University of Nebraska Medical Center, Omaha, NE 68198-4388, USA; \\ ellen.duysen@unmc.edu (E.D.); aaron.yoder@unmc.edu (A.M.Y.) \\ * Correspondence: aramos@unmc.edu; Tel.: +1-402-559-2095
}

Received: 31 March 2019; Accepted: 30 April 2019; Published: 30 April 2019

\begin{abstract}
Cattle feedyards are a high-risk environment. They are characterized by high rates of occupational injuries and illnesses. As such, there is a clear need to address the health and safety of cattle feedyard workers. Therefore, the purpose of this cross-sectional study was to explore safety training practices and preferences in the cattle feeding industry. A survey of feedyard managers, feedyard safety trainers, and feedyard operators was conducted $(n=28)$. We found that only half of respondents had dedicated safety personnel; however, there was interest in a safety training program, conducted through short hands-on and in-person methods with materials available in English and Spanish. The majority of participants were also interested in a feedyard safety certification program. Participants reaffirmed the importance of partnering with industry and other stakeholders when conducting these types of programs. The results of this Phase 1-type translational research study will be used to guide the development of feedyard safety trainings and a corresponding recognition program for feedyards and feedyard workers as part of the "Improving Safety and Health of Cattle Feedyard Workers" project.
\end{abstract}

Keywords: feedyard safety; feedyard hazards; feedlots; agricultural health and safety; translational research; research-to-practice (r2p)

\section{Introduction}

Agriculture continues to be one of the most dangerous industries in the United States and across the world, characterized by high rates of occupational injuries and illnesses [1,2]. Cattle feedyards, a type of intensive animal feeding operation, are no exception. For example, the occupational fatality rate in the beef cattle ranching and farming industries including feedyards (North American Industry Classification System (NAICS) code, 11211) in 2017 was 151 fatalities per 100,000 workers, a rate six times higher than that of the agriculture, forestry, and fishing sector $(23.0 / 100,000)$ and 43 times higher than the rate of all industries combined $(3.5 / 100,000)$ [2]. The cattle sector had nearly $10 \%$ of all fatalities (57 of 581) reported in the agriculture, forestry, and fishing sector that year. A similar trend is found in the incidence of nonfatal occupational injuries and illnesses. For example, in 2017, hired workers in the beef cattle ranching and farming including feedyards had a "days away from work" rate of 316.3/10,000 workers, while the rate for the agriculture, forestry, and fishing sector overall was 170.5 and all industries combined was 89.4. The rate for nonfatal occupational injuries and illnesses in the beef cattle sector increased in 2017 compared to the previous three years from 254.2, 255.2, and 187.9 in 2014, 2015, and 2016, respectively, while rate for all industries remained stable [3]. 
The cattle feeding industry is concentrated in the Midwestern part of the United States and in Texas. In fact, in the first quarter of 2018, the seven-state region including North Dakota, South Dakota, Nebraska, Kansas, Missouri, Iowa, and Minnesota had 586 cattle feedyards and 7019 feedyard employees. This represents 53\% of all feedyards and $49 \%$ of feedyard workers in the U.S. The majority of the feedyards and employees in this region were located in Nebraska (251 feedyards; 2943 employees) and Kansas (141 feedyards; 2968 employees) [4], and it is estimated that the workforce consists of about $50 \%$ immigrant workers, many of whom have low levels of formal education and limited English language proficiency [5]. Agricultural injury surveillance data from this same seven state region found that $37.7 \%$ of all reported injuries resulted from contact with livestock [6].

\subsection{Translational Research}

Because there are high rates of occupational injury in the cattle feeding industry, few evidence-based prevention programs available, and a myriad of diverse challenges in agriculture, a better preventative system is necessary. Safety resources on cattle feedyards may be scarce and dependent on the size of the operation. For example, larger feedyards may have more access to specific safety training programs or dedicated safety personnel who are charged with managing employee personal protective equipment, providing safety training and appropriate certifications, and reporting injuries and near-misses. However, employees on smaller operations may have to be able to work multiple feedyard positions such as cowboy, in the hospital or sick pen, processing cattle, or delivering feed to the animals, whereas employees in larger feedyards may have the opportunity to specialize in a certain area of the operation. Clearly, the size of the operation may make a difference. Eliminating all of the hazards would be the most effective way to reduce such injuries, but that is not feasible. Therefore, finding ways to reduce or mitigate the hazards is important, and one of the possible strategies is more effective worker training [7] that takes into account the capacity of the feedyard and engages the end users in the design of the program $[8,9]$. This is particularly important for ensuring the specific cultural and linguistic needs of the immigrant workforce are met [9].

There is a clear need for translational research to address the health and safety of cattle feedyard workers. Translational research, also known as research to practice (r2p), is vital to preventing occupational fatalities, injuries, and illnesses among workers [10]. The National Institute for Occupational Safety and Health (NIOSH) has recognized that bridging the gap between research and practice is vital because it takes many years for a health or safety research innovation to be widely used in practice [11,12], and many innovations never make it into practice.

Schulte and colleagues (2017) developed a conceptual framework for translational research in occupational safety and health, which consists of four phases: (1) development, (2) testing, (3) institutionalization, and (4) evaluation [13]. Phase 1 (Development) explores a potential intervention, including "proof of concept" testing, dosage, and possible strategies to scale up. This phase is important to ensuring that the concept is of interest to the targeted group of end users since one of the main reasons agricultural producers do not participate in training is that it does not respond to their needs [14]. Phase 2 (Testing) looks at whether a pilot intervention is feasible and effective in the workplace. Phase 3 (Institutionalization) focuses on whether the intervention actually works and assesses dissemination and implementation across a broad range of workplace settings. Finally, phase 4 (Evaluation) measures the outcomes and impact of the intervention [13].

\subsection{Responding to the Need}

In response to the identified need, the Central States Center for Agricultural Safety and Health (CS-CASH), a NIOSH-funded Agricultural, Forestry, and Fishing Center developed a research project entitled "Improving Safety and Health of Cattle Feedyard Workers". The long-term goal of this project is to reduce the high rates of injury, illness, and death in the cattle feeding industry. A Feedyard Safety Advisory Board consisting of feedyard representatives and related industry partners was created to serve as a sounding board, providing feedback and recommendations to guide this project. A health 
and safety program model will also be created as part of this project and tested in collaboration with industry and insurance partners as well as other feedyard stakeholders. However, before developing this program, a better understanding about the industry and its training needs was paramount. Therefore, the purpose of this study was to explore safety training practices and preferences in the cattle feeding industry. We employed a similar approach as Caffaro and colleagues (2017) to assess types and organization of training [9]. We expected that there would be differences in practices and preferences based on feedyard size. Below are results of a phase 1 translational research study, which will help guide the development of feedyard safety training to be used as part of the research intervention and eventually be available to feedyard safety professionals to implement in practice.

\section{Materials and Methods}

\subsection{Participants}

Participants were located in the Midwestern part of the United States and included feedyard managers, feedyard safety trainers, and feedyard operators that were members of the Nebraska Cattlemen, an industry specific organization. Because of the survey dissemination strategy using an industry organization and an insurance company partner, there were no specific individual participant inclusion criteria. Any beef cattle operation in the area was eligible to participate.

\subsection{Procedures}

A 33-question survey was developed, in part based off previous work by Ramos et al. (2018) [5] to assess current training practices and preferences in the cattle feeding industry. The survey was vetted by the project's Feedyard Safety Advisory Board and each item was discussed to ensure it was relevant to the target audience. The survey was later refined in collaboration with several industry partners, including the Nebraska Cattlemen, in early 2018. Surveys were disseminated online by the Nebraska Cattlemen to their members, and hard copies were disseminated by an insurance company partner to their clients in the Midwest region (primarily feedyard clients located in Nebraska), and thus there may have been multiple ways that feedyards could have found out about the study. Surveys assessed current training practices and preferred training methodologies, as well as demographic characteristics of the feedyard operation. Surveys took approximately 10 minutes to finish, and all surveys were completed between July-August 2018. Because the data from this survey will be used to improve the quality of the training materials that are developed, no institutional review board approval was necessary.

\subsection{Measures}

\subsubsection{Registered Capacity}

The registered capacity of the feedyard, the maximum number of cattle that an operator can feed at any one time during the year, was recorded as a continuous variable and later categorized into small (less than 1000 head), medium (between 1000-7999 head), large (8000-32,000 head), and extra-large (over 32,000 head) based on United States Department of Agriculture (USDA) categories and those used in a previous study [5,15]. We believed that size of the operation may influence not just the employer's ability to provide safety training to workers but also their interest in specific methodologies and topics [14].

\subsubsection{Dedicated Safety Personnel}

Participants were asked if they have a person dedicated to safety on the feedyard. Responses were dichotomous, yes or no. If a participant responded that they did not have any dedicated safety personnel, they were then asked about the barriers to having such a position at their operation. 


\subsubsection{Training}

Participants were asked about the current training methods that they employed as well as their preferred training methods. Response options included: in-person/classroom, videos, shadowing or watching another worker, hands-on practice, and written materials. Participants could choose all that applied. Participants were asked to rate how much time they spent training their workers on animal health, animal safety, worker health, and worker safety. Response options include a little (less than 1 hour/year), some (between 1-52 hours/year), and a lot (more than 52 hours/year). Participants were asked about their preferred length of training, and response options included: less than 1 hour, 1-2 hours, 3-4 hours, or a full day. They were also asked about their preferred frequency of training, and response options included: weekly, biweekly, monthly, quarterly, seasonally, or annually. The need for training immigrant workers was assessed, as well as what languages were spoken by the immigrant workforce. Finally, participants were asked about their interests for safety training and materials should they become available, and response options included regulatory compliance training (e.g., compliance with Occupational Safety and Health Administration [OSHA] regulations); hiring a certified safety professional; materials to develop a safety professional internally; sending an employee for training to become a safety professional; cardiopulmonary resuscitation (CPR)/first aid; online safety training; materials for face-to-face training; and a feedyard safety certification program. Again, participants could choose all that applied.

\subsubsection{Information Sources}

Participants were asked about where they look for safety information. Response options included Extension, insurance companies, online (e.g., YouTube, National Ag Safety Database), organizations (e.g., Nebraska Cattlemen, USDA, or agricultural safety centers), veterinarians, animal health companies, consultants, or other feedyards. There was also an option to list any other source of information. Participants could choose all that applied.

\subsection{Analyses}

This descriptive analysis uses data from only feedyard operations or feedyards that also had a cow-calf operation. Facilities that were strictly cow-calf operations were dropped from this analysis. Descriptive statistics were used to describe the characteristics of participant feedyards. Chi square tests were used to identify significant bivariate relationships between registered capacity and having dedicated safety personnel. SPSS version 25 was used for all analyses.

\section{Results}

A total of 47 surveys were completed; however, only those that were classified as feedyards or feedyard/cow-calf operations are included in the analyses $(n=28)$, representing $11.2 \%$ of the total number of feedyards in Nebraska. Respondents that were strictly cow-calf operations were discarded. Respondents were located mainly in Nebraska, but some also had operations in Kansas. The average registered capacity of feedyard participants was 15,206 head, but capacity ranged between 200 and 113,750 head. The average number of full-time workers was 15, but responses ranged between 1 and 65 workers (Table 1 ). 
Table 1. Characteristics of participant feedyards.

\begin{tabular}{ccc}
\hline Variable & $N(\%)$ & $M(S D)$ \\
\hline Registered Capacity of Feedyard (head) & & $15,026.8(25,870.1)$ \\
\hline Feedyard Size & & \\
Small (Less than 1000 head) & $7(25.0)$ & \\
Medium (1000-7999 head) & $8(28.6)$ & \\
Large (8000-32,000 head) & $9(32.1)$ & \\
Extra Large (Over 32,000 head) & $4(14.3)$ & \\
\hline Employees & & $14.9(20.2)$ \\
Full-Time & & $0.40(1.4)$ \\
Part-Time & $14(50.0)$ & \\
\hline Have Dedicated Safety Personnel & \\
\hline Provide Safety Orientation to Employees & $23(82.1)$ & \\
\hline
\end{tabular}

\subsection{Dedicated Safety Personnel}

Half of feedyard participants had a person(s) dedicated to safety (Table 1). However, there was a significant difference in reporting having a staff member dedicated to safety based on feedyard size, $\chi^{2}=12.35, p=0.006$. Large and extra-large feedyards were more likely to report having dedicated safety personnel, but only one respondent had a full-time person dedicated to safety. Barriers that were identified by those who did not have dedicated safety personnel included cost of hiring, time, and the perception that such a position was not needed because of the small size of the operation.

\subsection{Training}

Nearly $93 \%$ of respondents provided some type of training to employees, and more than $82 \%$ of respondents provided safety instructions or training for newly hired employees (Table 1). Hands-on practice, in-person/classroom training, and shadowing or watching another worker were the most frequently cited methods of training (Table 2). Worker health was a less frequent training topic than animal health or safety and worker safety.

Most respondents had a strong preference for hands-on training (Table 2). Nearly $60 \%$ of participant feedyards preferred trainings that were less than 1-hour. About half of participant feedyards preferred weekly $(21.4 \%)$ or monthly $(28.6 \%)$ trainings. One-third of participant feedyards reported a need to train immigrant workers, and Spanish was the only foreign language reported being spoken by these workers.

Table 2. Current and preferred safety training methods.

\begin{tabular}{|c|c|c|c|c|c|}
\hline Registered Capacity & $\begin{array}{c}\text { In-Person/ } \\
\text { Classroom } \\
\text { Training } \\
N(\%)\end{array}$ & $\begin{array}{l}\text { Videos } \\
N(\%)\end{array}$ & $\begin{array}{c}\text { Shadowing } \\
\text { Other } \\
\text { Workers } \\
N(\%)\end{array}$ & $\begin{array}{l}\text { Hand-On } \\
\text { Training } \\
N(\%)\end{array}$ & $\begin{array}{c}\text { Written } \\
\text { Materials } \\
N(\%)\end{array}$ \\
\hline \multicolumn{6}{|l|}{ Current training methods } \\
\hline Small (Less than 1000 head) & $2(28.6)$ & $1(14.3)$ & $1(14.3)$ & $3(42.9)$ & $1(14.3)$ \\
\hline Medium (1000-7999 head) & $5(62.5)$ & $1(12.5)$ & $4(50.0)$ & $6(75.0)$ & $1(12.5)$ \\
\hline Large (8000-32,000 head) & $6(66.7)$ & $3(33.3)$ & $5(55.6)$ & $6(66.7)$ & $5(55.6)$ \\
\hline Extra Large (Over 32,000 head) & $2(50.0)$ & $1(25.0)$ & $3(75.0)$ & $4(100.0)$ & 0 \\
\hline \multicolumn{6}{|l|}{ Preferred training methods } \\
\hline Small (Less than 1000 head) & $2(28.6)$ & 1 (14.3) & $1(14.3)$ & $3(42.9)$ & $1(14.3)$ \\
\hline Medium (1000-7999 head) & $5(62.5)$ & 0 & $3(37.5)$ & $8(100.0)$ & $1(12.5)$ \\
\hline Large (8000-32,000 head) & $5(55.6)$ & $2(22.2)$ & $6(66.7)$ & $8(88.9)$ & $3(33.3)$ \\
\hline Extra Large (Over 32,000 head) & $1(25.0)$ & $1(25.0)$ & $2(50.0)$ & $4(100.0)$ & 0 \\
\hline
\end{tabular}


Over half of respondents (53.6\%) were interested in a feedyard safety certification program, and $46.3 \%$ were interested in materials for conducting face-to-face training. Participants were also interested in regulatory compliance training that met OSHA compliance requirements (39.3\%). Hiring a certified safety professional was of the least interest.

\subsection{Information Sources}

Most commonly used sources for obtaining health and safety information reported by participant feedyards included: industry organizations $(57.1 \%)$, consultants $(53.6 \%)$, insurance companies $(50 \%)$, veterinarians (46.4\%), animal health companies (39.3\%), and University Extension programs (28.6\%).

\section{Discussion}

This study assessed safety training needs in the cattle feeding industry in the Midwestern part of the United States. Although others have explored safety training needs and practices among immigrant cattle feedyard workers [5], this is the first study to solicit information directly from feedyard employers. Based on our findings, there was a clear interest in safety training and the development of a feedyard certification program. Hands-on training was the preferred method for training among all sized operations; however, among large and extra-large operations, shadowing other workers ranked second, but among small and medium size operations in-person or classroom training ranked second. There was little interest in hiring or developing a "safety person", with participant feedyards citing cost, time, and size of operation as reasons why a position like this was not needed. In accordance with task specialization and resource availability of larger operations, large and extra-large feedyards were more likely to have dedicated safety personnel than smaller operations. Prior research has linked business size to accessibility and use of safety resources, with smaller organizations less likely to have access to these types of resources [16]. Most participant feedyards provided some type of training to their employees, but it was often not standardized.

Consistent training is imperative for all workers, particularly those in high-risk industries such as agriculture. Research has shown that regular task-specific training can reduce the likelihood of suffering an occupational injury [17]. Participant feedyards provided training mainly through hands-on practice, in-person/classroom training, and shadowing another worker, which is consistent with what has been found among dairy operations [18]; moreover, participant feedyards had a strong preference for hands-on training. Such training aligns with previous research that indicated that more engaging training methods resulted in greater knowledge acquisition and improvements in safety behaviors [7].

These findings, along with input from the Feedyard Safety Advisory Board, have led to the development of the "Feedyard 15" program, which seeks to address the top fifteen hazards on cattle feedyards. The Feedyard 15 includes the following topics:

1. All-Terrain Vehicle (ATV)/Utility Task Vehicle (UTV)

2. Bunker Silos

3. Cattle Handling/Stockmanship

4. Chemical Hazards

5. Electrical Hazards

6. Emergency Response

7. Extreme Weather

8. Feedmill Safety

9. Horsemanship

10. Machine Shop Hazards

11. Manure Lagoons

12. Mobile Equipment/Autos

13. Processing Cattle

14. Slips, Trips, and Falls

15. Tractors/Loaders 
As part of the "Improving Safety and Health of Cattle Feedyard Workers" project, training modules will be developed for each of the fifteen topics that have been identified. These modules will be tested in safety trainings with intervention feedyard workers and managers. The modules may be used in both hands-on and in-person trainings, thereby addressing the preferences of operations of all sizes and in particular those of small and medium sized operations, which may have less access to training resources. All modules are designed to be completed in less than one hour and will be available in both English and Spanish. Feedyards that participate in the program will be expected to complete one module per month. All feedyards and each worker will be able to obtain a certificate of recognition upon completion of twelve modules. Workers will also be eligible for Feedyard 15 branded gear including t-shirts, hats, personal protective equipment, and tools as incentives. Extrinsic motivators have been found to increase satisfaction and participation in such programs [19] plus these items will serve as cues to action to motivate safety behaviors. Upon the completion of the research study, all training modules will be available free of charge to the public. Although the hope is that these modules will increase safety knowledge and positively modify safety behaviors, we recognize that having a community of support (e.g., other workers or family members) to reinforce what was learned and motivate safety behaviors is critical $[8,20]$.

Another important finding from this study included the reaffirmation that the program should be conducted in partnership with industry organizations such as the Nebraska Cattlemen, insurance companies, and professionals such as veterinarians. Because these groups and professionals are connected with feedyards who would be the end-users of a safety training program, they can provide valuable insights to guide the development of the program $[9,21]$. These groups also already have the trust of feedyard operators, which may help with the active dissemination and diffusion of the program while promoting the importance of safety training within the industry.

This study had a number of limitations to note. First, data are cross-sectional and represent a snapshot in time. Second, we had a limited sample size that was drawn from one region of the country. Third, there may have been self-selection bias present, and those not interested in safety may have chosen not to participate. Finally, data are based on self-reports. There is the risk that the person who responded on behalf of the feedyard may have answered based on their personal experience and not necessarily objectively as a representative of the organization. These limitations could be mitigated by tracking training practices and preferences over time, obtaining data from other parts of the country where there is a high concentration of feedyards such as Texas, using trained observers to record current training methodologies used on feedyards, and ensuring that the person responding to the survey is responding as a representative of their organization.

Future research should explore the acceptability of training topics within the Feedyard 15; the feasibility and efficacy of training methodologies, program dosage, motivators, and barriers to use among feedyards; cost effectiveness of training components; and finally, the effectiveness and impact of the Feedyard 15 on health and safety outcomes including workers' compensation. Studies may also evaluate the effectiveness of the dissemination across the industry [22].

In conclusion, working on a cattle feedyard can be risky to one's health and safety, as proven by the occupational injury and illness data. Appropriate job-related training can be important to mitigating one's risk, as well as the associated risk to the feedyard employer. By using a translational research methodology, the time it takes for safety and health innovations such as the Feedyard 15 to be used in practice may be reduced. Furthermore, through the translational process, the program will have been refined to a point where it is feasible, acceptable, and well-suited to meet the needs of workers and employers within the cattle feeding industry, thereby making uptake and impact much more likely.

Author Contributions: Conceptualization, A.K.R., E.D., and A.M.Y.; methodology, A.K.R. and A.M.Y.; formal analysis, A.K.R.; writing-original draft preparation, A.K.R.; writing-review and editing, A.K.R., E.D., and A.M.Y.; supervision, A.M.Y.; project administration, A.K.R., E.D., A.M.Y.; funding acquisition, E.D. and A.M.Y. 
Funding: This research was funded by the National Institute for Occupational Safety and Health (NIOSH) grant number U54 OH010162 through the Central States Center for Agricultural Safety \& Health.

Acknowledgments: We would like to thank Mike Keenan with Gallagher Insurance, Risk Management and Consulting for his invaluable industry insights and continued partnership with this project. We also recognize the Nebraska Cattlemen for their support in disseminating the survey to their members and providing an opportunity to work more closely together.

Conflicts of Interest: The authors declare no conflict of interest. The funders had no role in the design of the study; in the collection, analyses, or interpretation of data; in the writing of the manuscript, or in the decision to publish the results.

\section{References}

1. International Labour Organization. Safety and Health in Agriculture. International Labour Organization: Geneva. Available online: https://www.ilo.org/wcmsp5/groups/public/---ed_dialogue/---sector/documents/ normativeinstrument/wcms_161135.pdf (accessed on 16 April 2019).

2. Bureau of Labor Statistics. Census of Fatal Occupational Injuries; U.S. Department of Labor: Washington, DC, USA, 2017. Available online: http://www.bls.gov/iif/oshcfoi1.htm (accessed on 19 March 2019).

3. Bureau of Labor Statistics. Occupational Injury/Illness and Fatal Injuries Profiles, 2017; U.S. Department of Labor: Washington, DC, USA, 2017. Available online: http://data.bls.gov/gqt/ (accessed on 19 March 2019).

4. Bureau of Labor Statistics. Quarterly Census of Employment and Wages; U.S. Department of Labor: Washington, DC, USA, 2017. Available online: http://www.bls.gov/cew/apps/data_views/data_views.htm (accessed on 19 March 2019).

5. Ramos, A.K.; Carlo, G.; Grant, K.M.; Bendixsen, C.; Fuentes, A.; Gamboa, R. A preliminary analysis of immigrant cattle feedyard worker perspectives on job-related safety training. Safety 2018, 4, 37. [CrossRef]

6. Jadhav, R.; Achutan, C.; Haynatzki, G.; Rajaram, S.; Rautiainen, R. Injury risk factors to farm and ranch operators in the Central United States. Am. J. Ind. Med. 2017, 60, 889-899. [CrossRef] [PubMed]

7. Burke, M.J.; Sarpy, S.A.; Smith-Crowe, K.; Chan-Serafin, S.; Salvador, R.O.; Islam, G. Relative effectiveness of worker safety and health training methods. Am. J. Public Health 2006, 96, 315-324. [CrossRef] [PubMed]

8. Caffaro, F.; Micheletti Cremasco, M.; Bagagiolo, G.; Vigoroso, L.; Cavallo, E. Effectiveness of occupational safety and health training for migrant farmworkers: A scoping review. Public Health 2018, 160, 10-17. [CrossRef] [PubMed]

9. Cafffaro, F.; Bagagiolo, G.; Micheletti Cremasco, M.; Cavallo, E. Participatory ergonomic design of a safety training tool for migrant workers in agriculture. Chem. Eng. Trans. 2017, 58, 25-30.

10. National Institute on Occupational Safety and Health. Research to Practice ( $2 p$ ); National Institute on Occupational Safety and Health: Morgantown, WV, USA, 2018. Available online: https://www.cdc.gov/ niosh/r2p/about.html (accessed on 19 March 2019).

11. Balas, E.A.; Boren, S.A. Yearbook of Medical Informatics: Managing Clinical Knowledge for Health Care Improvement; Schattauer Verlagsgesellschaft mbH: Stuttgart, Germany, 2000.

12. Westfall, J.M.; Mold, J.; Fagnan, L. Practice-based research—“Blue Highways” on the NIH roadmap. JAMA 2007, 297, 403-406. [CrossRef] [PubMed]

13. Schulte, P.A.; Cunningham, T.R.; Nickels, L.; Felknor, S.; Guerin, R.; Blosser, F.; Chang, C.; Check, P.; Eggerth, D.; Flynn, M.; et al. Am. J. Ind. Med. 2017, 60, 1011-1022. [CrossRef] [PubMed]

14. Lioutas, E.D.; Tzimitra-Kalogianni, I.; Charatsari, C. Small ruminant producers' training needs and factors discouraging participation in agricultural education/training programs. Livest. Res. Rural Dev. 2010, 22.

15. U.S. Department of Agriculture, Animal and Plant Health Inspection Service. Feedlot 2011. Part I: Management Practices on U.S. Feedlots with a Capacity of 1000 or More Head. United States Department of Agriculture: Fort Collins, CO, USA, 2013. Available online: https://www.aphis.usda.gov/animal_health/ nahms/feedlot/downloads/feedlot2011/Feed11_dr_PartI.pdf (accessed on 17 August 2018).

16. Sinclair, R.C.; Cunningham, T.R. Safety activities in small business. Saf. Sci. 2014, 64, 32-38. [CrossRef] [PubMed]

17. Román-Muñiz, I.N.; Van Metre, D.C.; Garry, F.B.; Reynolds, S.J.; Wailes, W.R.; Keefe, T.J. Training Methods and Association with Worker Injury on Colorado Dairies: A Survey. J. Agromed. 2006, 11, 19-26. [CrossRef] [PubMed] 
18. Sorge, U.S.; Cherry, C.; Bender, J.B. Perception of the importance of human-animal interaction on cattle flow and worker safety on Minnesota dairy farms. J. Dairy Sci. 2014, 97, 4632-4638. [CrossRef] [PubMed]

19. Rovai, M.; Carroll, H.; Foos, R.; Erickson, T.; Garcia, A. Dairy tool box talks: A comprehensive worker training in dairy farming. Front Public Health 2016, 4, 136. [CrossRef] [PubMed]

20. Unay Gailhard, I.; Bavorova, M.; Pirscher, F. Adoption of agri-environmental measures by organic farmers: The role of interpersonal communication. J. Agric. Educ. Ext. 2015, 21, 127-148. [CrossRef]

21. Ramos, A.K.; Trinidad, N.; Correa, A.; Rivera, R. Partnering for health with Nebraska's Latina immigrant community using design thinking process. Prog. Community Health Partnersh. 2016, 10, 311-318. [CrossRef] [PubMed]

22. Dugan, A.G.; Punnett, L. Dissemination and implementation research for occupational safety and health. Occup. Health Sci. 2017, 1, 29-45. [CrossRef] [PubMed]

(C) 2019 by the authors. Licensee MDPI, Basel, Switzerland. This article is an open access article distributed under the terms and conditions of the Creative Commons Attribution (CC BY) license (http://creativecommons.org/licenses/by/4.0/). 